\title{
ARCHIMEDEAN EXTENSIONS OF DIRECTED INTERPOLATION GROUPS
}

\author{
A. M. W. GLass
}

P. F. Conrad has obtained some properties of archimedean extensions ( $a$-extensions) of lattice ordered groups ( $l$-groups). In particular, Conrad proved that every abelian $l$-group has an $\mathscr{A}$-closure (an abelian $a$-extension which has no proper abelian $a$-extension). D. Khuon proved that every $l$-group has an $a$-closure (an $a$-extension which has no proper $a$-extension). Using a slightly different definition, Conrad and Bleier defined an $a^{*}$-extension of an $l$-group and proved that every abelian $l$-group has an $a^{*}$-closure and every archimedean $l$-group has a unique $a^{*}$-closure. These results have been extended to another class of $l$-groups by Glass and Holland (unpublished).

The purpose of this paper is to extend the $l$-group results to the class of directed interpolation groups. The obvious definitions give rise to some negative results; the situation

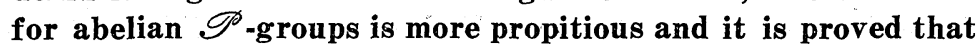
any such group has an $\mathscr{A}$-closure in this class. However, taking less direct definitions of $a$-extensions and $a^{*}$-extensions gives $\mathscr{A}$-closures and $\mathscr{A}{ }^{*}$-closures in restricted classes of abelian directed interpolation groups.

It is assumed that the reader is familiar with [1], [2], [3], [4], and [5]

1. Definitions and notation. Throughout this paper, additive notation will be used for all groups, abelian or not. $\subset$ will denote strict containment and $\subseteq$ will denote strict containment or equality. On will denote the collection of all ordinals.

If $A$ is a p.o. set and $\alpha, \beta \in A$, then $\alpha \| \beta$ will stand for $\alpha \nsubseteq \beta$ and $\beta \geqq \alpha$.

If $G$ is a group and $X \subseteq G,\langle X\rangle$ will denote the subgroup of $G$ generated by $X$. If $G$ and $H$ are groups $G \oplus H$ will denote the cartesian sum of $G$ and $H$. If $G$ and $H$ are p.o. groups, then $G \vec{\oplus} H$, the lexicographic sum of $G$ over $H$, is the group $G \oplus H$ ordered by: $(g, h)>0$ if and only if $g>0$ (in $G$ ) or $g=0$ and $h>0$ (in $H$ ). If $\left\{G_{\alpha}: \alpha \in A\right\}$ is a family of p.o. groups, then $\Pi\left\{G_{\alpha}: \alpha \in A\right\}\left(\Sigma\left\{G_{\alpha}: \alpha \in A\right\}\right)$ will denote the cartesian product (sum) of the family of groups $\left\{G_{\alpha}: \alpha \in A\right\}$ ordered by: $g \geqq 0$ if $g_{\alpha} \geqq 0$ (in $G_{\alpha}$ ) for all $\alpha \in A ; \Pi^{*}\left\{G_{\alpha}: \alpha \in A\right\}$ is the same group as above but ordered by: $g>0$ if and only if $g_{\alpha}>0$ (in $G_{\alpha}$ ) for all $\alpha \in A$. 
If $G$ is a p.o. group, $G^{+}$will denote the positive cone of $G=$ $\{g \in G: g \geqq 0\}$ and $G^{*}$ will denote the strictly positive cone of $G=$ $\{g \in G: g>0\} . R(Z)$ will denote the additive o-group of reals (integers) but $\boldsymbol{R}^{+}\left(\boldsymbol{Z}^{+}\right)$will denote the strictly positive reals (integers).

Let $G$ be a p.o. group. The partial order is said to be dense if and only if for all $g, h \in G$, if $g<h$ there exists $f \in G$ such that $g<f<h$. The partial order satisfies the interpolation property if whenever $g, h, f, k \in G$ and $g, h \leqq f, k$, there exists $x \in G$ such that $g, h \leqq x \leqq f, k$. A p.o. group satisfying the interpolation property is called an interpolation group. A directed interpolation group $G$ in which $f \vee g$ (or $f \wedge g$ ) exists only when $f \leqq g$ or $g \leqq f$ is said to be an antilattice. A directed group $G$ such that for all $f, g, h, k \in G$ whenever $g, h<f, k$, there exists $x \in G$ such that $g, h<x<f, k$ is called a tight Riesz group. Let $\bar{P}=\left\{g \in G: g \in G^{+}\right.$or $g$ is pseudo-positive $\}$ and let the cone of $\leqq$ be $\bar{P}$. If $G$ has no pseudo-identities, then $\leqq$ is said to be a compatible tight Riesz order for $(G, \leqq)$.

Let $A$ be a partially ordered set. For each $\alpha \in A$. Let $R_{\alpha}$ be a partially ordered abelian group. Let $K$ be the cartesian product of $\left\{R_{\alpha}: \alpha \in A\right\}$. The set of all $k \in K$ such that $\left\{\alpha \in A: k_{\alpha} \neq 0\right\}$ satisfies the ascending chain condition (in $A$ ) forms abelian group which is denoted by $V\left(A, R_{\alpha}\right)$. For each $v \in V\left(A, R_{\alpha}\right)$, let $M(v)=\left\{\alpha \in A: v_{\alpha} \neq 0\right.$ and $v_{\beta}=0$ for all $\beta \in A$ such that $\left.\beta>\alpha\right\} . \quad V\left(A, R_{\alpha}\right)$ is a partially ordered abelian group under the ordering: $v>0$ if and only if $v_{\alpha}>0$ for all $\alpha \in M(v)$.

2. A naive approach to $a$-extensions of directed interpolation groups. Let $H$ be a directed interpolation group and let $G$ be a subgroup of $H . \quad \mathrm{G}$ is said to be an interpolation subgroup of $H$ if and only if for all $x, y \in G$ and $z, t \in H, x, y \leqq z, t$ implies there exists $g \in$ $G$ such that $x, y \leqq g \leqq z, t$. Note that this condition is equivalent to: $x, y \in G$ and $z, t \in H$ and $x, y \geqq z, t$ imply there exists $g \in G$ such that $x, y \geqq g \geqq z, t$.

It should be observed that if $H$ is an $l$-group and $G$ an interpolation subgroup of $H$, then $G$ is an $l$-subgroup of $H$. However, let $H=V\left(A, R_{\alpha}\right)$ and $G=V\left(B, R_{\beta}\right)$ where $A=\{1, \overline{2}, \overline{3}\}$ ordered by: $\overline{1}, \overline{2}>\overline{3}$ and $\overline{1}|| \overline{2}, B=\{\overline{1}, \overline{2}\}$ and $R_{\alpha}=R$ for all $\alpha \in A$. $H$ is a directed interpolation group and $G$ is an l-group under the induced ordering. However, $G$ is not an interpolation subgroup of $H$ as $(0,0,1) \leqq(1,0,0),(0,1,0)$ but there is no $g \in G$ such that $(0,0,1) \leqq g \leqq(1,0,0),(0,1,0)$.

$h_{1}, h_{2} \in H^{+}$are said to be a-equivalent if and only if there exist $n_{1}, n_{2} \in Z^{+}$such that $h_{1} \leqq n_{2} h_{2}$ and $h_{2} \leqq n_{1} h_{1}$. $\quad H$ is an a-extension of $G$ if and only if every $h \in H^{+}$is $a$-equivalent to some $g \in G^{+}$and $G$ is 
an interpolation subgroup of $H$. This coincides with the definition given in [2] for $l$-groups and is a natural extension to directed interpolation groups. Notice that if $K$ is an $a$-extension of $H$ and $H$ is an $a$-extension of $G$, then $K$ is an $a$-extension of $G$. Using the method of [2] and the results of [4] and [5] it is easy to see that $H$ is an $a$-extension of $G$ if and only if there is $a(1: 1)$ map of the convex $d$-subgroups of $G$ onto those of $H$ which preserves inclusion and maps prime subgroups of $G$ (polars of $G$ ) to prime subgroups of $H$ (polars of $H$ ).

$G$ is a-closed if and only if $G$ has no proper a-extension. In view of [2] and [6], we cannot hope for there to be a unique $a$-closure of a directed interpolation group but we can try to prove that any path of proper $a$-extensions eventually terminates. The next two theorems shatter this dream-the second gives a path of proper $a$-extensions of a certain class of abelian directed interpolation groups which cannot be closed up; the first, more dramatically, proves that no path of $a$-extensions of $\boldsymbol{R}$ can ever be closed up.

THEOREM A. Any a-extension (in the class of directed interpolation groups) of a dense antilattice is a dense antilattice. Consequently, no dense antilattice other than $\{0\}$ has an a-closure and no abelian dense antilattice other than $\{0\}$ has an a-closure in the class of abelian directed interpolation groups.

Proof. It is easy to see that any $a$-extension of an antilattice is an antilattice. Suppose $G$ is a dense directed interpolation group and that $H$ is an $a$-extension of $G$. We prove that $H$ is dense.

Assume that $0<h \in H$ and that there is no $k \in H$ such that $0<k<h$. Since $G$ is dense, $h \notin G$. We first show that, under these hypotheses, some multiple of $h$ belongs to $G$. Let $g$ be $a$-equivalent to $h$ and assume that $m \in \boldsymbol{Z}^{+}$-is the least such that $m g \geqq h$. Then $g, h \geqq 0, h-(m-1) g$. Since $H$ is an interpolation group, there exists $k \in H$ such that $g, h \geqq k \geqq 0, h-(m-1) g$. Thus $h \geqq k \geqq 0$ and so $h=k$ by the hypothesis and the choice of $m$. Consequently, $g \geqq h$. Let $n \in \boldsymbol{Z}^{+}$be least such that $n h \geqq g$ and let $p \in \boldsymbol{Z}^{+}$be greatest such that $g \geqq p h$. Now $p \leqq n$ and, by hypothesis, $p \neq n$. Hence $0, g-(n-1) \leqq g-g h, h$ and, as before, $h \leqq g-p h$, a contradiction. Thus $n h=g$ for some $\mathrm{n} \in \boldsymbol{Z}^{+}$and $g \in G^{+}$. Choose $g \in G^{+}$so that $n$ is minimal. Since $G$ is dense, there exists $g^{\prime} \in G$ such that $0<g^{\prime}<g=n h$. Let $p \in \boldsymbol{Z}^{+}$be least such that $g^{\prime} \leqq p h$. Then $p \leqq n$ and $g^{\prime}-(p-1) h, 0 \leqq g^{\prime}, h$. Hence there exists $k \in H$ such that $g^{\prime}-(p-1) h, 0 \leqq k \leqq g^{\prime}, h$. By hypothesis, $k=h$ and so $h \leqq g^{\prime}$. Let $q \in \boldsymbol{Z}^{+}$be greatest such that $q h \leqq g^{\prime}$. Thus $q \leqq p$. If $q<p$, then $q^{\prime}-(p-1) h, 0 \leqq g^{\prime}-q h, h$ and, as before, $h \leqq g^{\prime}-q h$. It follows that $(q+1) h \leqq g^{\prime}$, a contradic- 
tion. Consequently, $q=p$ and $g^{\prime}=p h$. By the choice of $n, p=n$. Hence $g^{\prime}=p h=n h=g$, a contradiction.

Finally suppose $K$ is an $a$-cloure of a dense antilattice $G \neq\{0\}$. Then $K$ is a dense antilattice. Let $L$ be any abelian trivially ordered group. It is immediate that $K \vec{\oplus} L$ is a dense antilattice which is a proper $a$-extension of $K$, a contradiction.

COROLlaRy A.1. $\quad \boldsymbol{R}$ has no a-closure in either the class of directed interpolation groups or the class of abelian directed interpolation groups.

THEOREM B. Suppose $A$ is a p.o. set and for each $\alpha \in A, R_{\alpha} \neq$ $\{0\}$ is a subgroup of either $\boldsymbol{R}$ or the trivially ordered additive group of reals. If $V\left(A, R_{\alpha}\right)$ is a directed interpolation group, then there exist $\left\{G_{\beta}: \beta \in O n\right\}$ such that $G_{0}=V\left(A, R_{\alpha}\right)$ and if $\lambda, \mu \in O n$ and $\lambda<\mu$, then $G_{\mu}$ is a proper a-extension of $G_{\lambda}$ in the class of abelian directed interpolation groups.

Proof. Let $B$ be a maximal totally ordered subset of $A$. If $\alpha_{0}$ is a minimal element of $B$ and $R_{\alpha_{0}} \cong Z$, let $S_{\alpha}=R_{\alpha}$ if $\alpha \neq \alpha_{0}$ and $S_{\alpha_{0}}=\boldsymbol{R}$. Then $W=V\left(A, S_{\alpha}\right)$ is a directed interpolation group by Teller's conditions (see [8]) and is an a-extension of $V=V\left(A, R_{\alpha}\right)$ so we may assume that if $B$ has a minimal element $\alpha_{0}, R_{\alpha_{0}}$ is a dense $o$-group or a subgroup of the trivially ordered additive group of reals. Let $\Gamma=A \cup\{\gamma\}$ where $\gamma \notin A$. $\Gamma$ is a p.o. set under the ordering: $\gamma_{1}<\gamma_{2}$ if and only if $\gamma_{1}<\gamma_{2}$ in $A$ or $\gamma_{1}=\gamma$ and $\gamma_{2} \in B$. Let $R_{\delta}=R_{\alpha}$ if $\delta \in A$ and $R_{r}$ be the trivially ordered additive group of reals. Then $U=V\left(\Gamma, R_{\delta}\right)$ is a directed interpolation group (by Teller's conditions) and an $a$-extension of $V$. Continuing in this fashion, the theorem is proved.

Even removing pseudo-identities does not help since $\boldsymbol{R} \boxplus^{*} \boldsymbol{R}$ is an $a$-extension of $\{(a, a): a \in \boldsymbol{R}\} \cong \boldsymbol{R}$ in the class of directed interpolation groups without pseudo-identities.

3. $a$-extensions of abelian $\mathscr{P}$-groups. Using the results of [3] and the methods of [2], the following generalizations of theorems of [2] are obtained:

THEOREM C.1. If $G$ is an abelian $\mathscr{P}$-group, then $G$ has an aclosure in the class of abelian $\mathscr{P}$-groups. If $H$ is any such a-closure of $G$ and $\triangle$ is a plenary subset of $C_{1}(G)$, then there exists an "l"isomorphism of $H$ into $V=V\left(\Delta, R_{\delta}\right)$.

THEOREM C.2. If $A$ is a p.o. set and $R_{\alpha}=\boldsymbol{R}$ for all $\alpha \in A$, then 
$V\left(A, R_{\alpha}\right)$ and $F\left(A, R_{\alpha}\right)$ are a-closed in the class of abelian $\mathscr{P}$-groups. Moreover, $F\left(A, R_{\alpha}\right)$ is an a-closure of $\Sigma\left(A, R_{\alpha}\right)$ in this class.

A p.o. group $G$ is said to be archimedean if and only if for all $f, g \in G, n f \leqq g$ for all $n \in Z$ implies $f=0$.

THEOREM D. If $G$ is an archimedean abelian $\mathscr{P}$-group, then so is any a-extension of $G$ in the class of abelian $\mathscr{P}$-groups.

Proof. Suppose that an abelian $\mathscr{P}$-group $H$ is an $a$-extension of the archimedean abelian $\mathscr{P}^{-g r o u p} G$ and $n h \leqq k$ for all $n \in Z(h, k \in H)$. If $h, k \geqq 0$, then there exist $f, g \in G$ which are $a$-equivalent to $h$ and $k$ respectively. It is easy to see that $n g^{\prime} \leqq f^{\prime}$ for all $n \in Z$ where $f^{\prime}, g^{\prime}$ are fixed multiples of $f, g$ respectively. Hence $g^{\prime}=0$ and so $g=0$. Thus $h=0$. Since $H$ is directed, it may be assumed that $k \geqq 0$. If $h \leqq 0$, the proof is the same as above so assume $h \| 0$. There exist $h_{1}, h_{2} \in H$ such that $h=h_{1}-h_{2}$ and $h_{1}, h_{2}$ are pseudo-disjoint. There exist $g_{1}, g_{2} \in G$ such that $g_{i}$ is $a$-equivalent to $h_{i}(i=1,2)$; say $m_{i} g_{i} \geqq h_{i}$ and $n_{i} h_{i} \geqq g_{i}(i=1,2)$. Now $n g_{1} \leqq n n_{1} h_{1}=n n_{1}\left(h+h_{2}\right) \leqq k+n n_{1} m_{2} g_{2}$ for all $n \in \boldsymbol{Z}$ and there exists $f \in G$ a-equivalent to $k$; say, $k \leqq p f, p \in \boldsymbol{Z}^{+}$. Now $n\left(g_{1}-n_{1} m_{2} g_{2}\right) \leqq p f$ for all $n \in Z$ and hence $g_{1}=n_{1} m_{2} g_{2}$. It follows that $h_{1} \leqq m_{1} g_{1}=m_{1} n_{1} m_{2} g_{2} \leqq m_{1} m_{2} n_{1} n_{2} h_{2}$ which is impossible since $h_{1}$ and $h_{2}$ are pseudo-disjoint. Thus $H$ is archimedean.

In [2], Conrad proved that every archimedean abelian $\mathscr{P}$-group (and hence every integrally closed abelian $\mathscr{P}$-group) is an l-group. Hence we have shown:

CoRollary D.1. Every a-extension of an archimedean l-group in the class of abelian $\mathscr{P}$-groups is an archimedean l-group.

In [2], the result was proved in the class of all $l$-groups not only abelian l-groups. Consequently, that result cannot be captured by the above proof. In view of Example 6.4 of [2], $a$-closures of archimedean abelian $\mathscr{P}$-groups are not unique.

K. M. van Meter has proved that every archimedean $\mathscr{P}$-group is an archimedean $l$-group and has proved that any $a$-extension of an archimedean $\mathscr{P}$-group in the class of $\mathscr{P}$-groups is an archimedean $\mathscr{P}$-group (see [9]) and so has proved a stronger theorem then Theorem D. The proof given here is more direct and was discovered independently and at the same time.

In view of Theorem $D$ and Theorem 3.1 of [1]:

Corollary D.2. Every archimedean (abelian) $\mathscr{P}$-group has a unique $a^{*}$-closure in the class of (abelian) $\mathscr{P}$-groups. 
4. Archimedean extensions of compatible tight Riesz groups. It is easy to see that $G$ is a tight Riesz group if and only if $G$ is a dense antilattice. By Theorem $\mathrm{A}$, any $a$-extension of a tight Riesz group is a tight Riesz group. We will restrict our attention to the class of directed interpolation groups without pseudo-identities and confine ourselves to those tight Riesz groups compatible with an $l$ group. Such partially ordered groups will be called acceptable tight Riesz groups. If $(G, \leqq)$ is an acceptable tight Riesz group, then $(G, \leqq)$ will be the $l$-group whose positive cone is the positive cone of $(G, \leqq)$ together with its pseudo-positive elements. Let $T=\{g \in G: g>0\}$. Then there exists $\left\{M_{\alpha}: \alpha \in A\right\}$ a collection of prime $l$-subgroups of $(G, \leqq)$ such that $T=G^{+} \backslash \cup\left\{M_{\alpha}: \alpha \in A\right\}$ (see Theorem 2.6 of [7]). Recall that if $(H, \leqq)$ is an $l$-group which is an $a$-extension of the $l$-group $(G, \leqq)$, then there is a $(1: 1)$ map $\phi$ of the prime $l$-subgroups of $(G, \leqq)$ onto the prime $l$-subgroups of $(H, \leqq)$ which preserves containment. Let $G$ be an abelian group and $X \subseteq G$. Let $\bar{G}$ be the divisible closure of $G$ and $\bar{X}=\left\{y \in G: n y \in X\right.$ for some $\left.n \in Z^{+}\right\}$. If $(G, \leqq)$ is an $l$-group, then $(\bar{G}$, $\leqq)$ is an $l$-group where $h \in \bar{G}^{+}$if and only if $n h \in G^{+}$for some $n \in Z^{+}$(i.e., $\bar{G}=\bar{G}^{+}$). Then $\bar{T}=\bar{G}^{+} \backslash \bigcup\left\{\bar{M}_{\alpha}: \alpha \in A\right\}$ is the strict positive cone of a compatible tight Riesz group $(\bar{G}, \leqq)$. Moreover, $(\bar{G}, \leqq)$ is an $a$-extension of $(G, \leqq)$ and there is a (1:1) map of the prime subgroups of $(G, \leqq)$ onto those of $(\bar{G}, \leqq)$ which preserves containment. Using these facts, we define what is meant by an $\mathscr{A}$ extension of an abelian acceptable tight Riesz group. In view of the above remarks, we need only concern ourselves with divisible abelian acceptable tight Riesz groups. If $(K, \leqq)$ is an abelian acceptable tight Riesz group and $(K, \leqq)$ the corresponding l-group, let $K^{+}=$ $\{k \in K: k \geqq 0\}$ and $T_{K}=\{k \in K: k>0\}$.

Let $(G, \leqq)$ and $(H, \leqq)$ be divisible abelian acceptable tight Riesz groups. $(H, \leqq)$ is an $\mathscr{A}$-extension of $(G, \leqq)$ if and only if $(H, \leqq)$ is an $\mathscr{A}$-extension of $(G, \leqq)$ and if $T_{G}=G^{+} \backslash \bigcup\left\{M_{\alpha}: \alpha \in A\right\}$ where each $M_{\alpha}$ is a prime $l$-subgroup of $(G, \leqq)$, then $T_{H}=H^{+} \backslash \bigcup\left\{N_{\alpha}: \alpha \in A\right\}$ where $N_{\alpha}$ is the prime $l$-subgroup of $(H, \leqq)$ corresponding to $M_{\alpha}$. It follows at once from the facts concerning $\mathscr{A}$-extensions of $l$-groups that:

THEOREM E. Every abelian acceptable tight Riesz group has an $\mathscr{A}$-closure (in the class of abelian acceptable tight Riesz groups) which is not necessarily unique. Moreover, any $\mathscr{A}$-extension of an archimedean abelian acceptable tight Riesz group is archimedean.

The last fact follows because $(G, \leqq)$ is archimedean if and only if $(G, \leqq)$ is. The same is not true for integrally closed since if $(G, \leqq)$ is $\boldsymbol{R} \boxplus^{*} \boldsymbol{R}$, then $(G, \leqq)=\boldsymbol{R} \boxplus \boldsymbol{R}$ is integrally closed whereas $(G, \leqq)$ is not. It should be observed that if $(H, \leqq)$ is an $\mathscr{A}$-extension of 
$(G, \leqq)$ where $(H, \leqq)$ and $(G, \leqq)$ are abelian divisible acceptable tight Riesz groups, then there is a (1:1) map of the convex $d$-subgroups of $(G, \leqq)$ onto the convex $d$-subgroups of $(H, \leqq)$ which preserves containment and for every $h \geqq 0$, there exists a $g \in G$ such that $g \geqq$ 0 and $g \leqq n h$ and $h \leqq m g$ for some $m, n \in \boldsymbol{Z}^{+}$.

An abelian acceptable tight Riesz group $(G, \leqq)$ will be called a good tight Riesz group if and only if $T_{G}=G^{+} \backslash \bigcup\left\{M_{\alpha}: \alpha \in A\right\}$ where each $M_{\alpha}$ is a closed prime subgroup of $(G, \leqq)$. As before, we need only consider divisible good tight Riesz groups.

Let $(H, \leqq)$ and $(G, \leqq)$ be divisible good tight Riesz groups. $(H, \leqq)$ is an $a^{*}$-extension of $(G, \leqq)$ if and only if $(H, \leqq)$ is an $a^{*}$-extension of $(G, \leqq)$ and if $T_{G}=G^{+} \backslash \bigcup\left\{M_{\alpha}: \alpha \in A\right\}$, then $T_{H}=H^{*} \backslash \bigcup\left\{N_{\alpha}: \alpha \in A\right\}$ where $N_{\alpha}$ is the closed prime $l$-subgroup of $(H, \leqq)$ corresponding to the closed prime $l$-subgroup $M_{\alpha}$ of $(G, \leqq)$.

ThEOREM F.1. Every good tight Riesz group has an a*-closure (in the class of good tight Riesz groups).

2. Every $\mathscr{A}$-extension (in the class of divisible abelian acceptable tight Riesz groups) of a good tight Riesz group $(G, \leqq)$ is a good tight Riesz group which is an $a^{*}$-extension of $(G, \leqq)$.

3. Every archimedean good tight Riesz group has a unique $a^{*}$ closure (in the class of good tight Riesz groups).

\section{REFERENCES}

1. R. Bleier and P. F. Conrad, The lattice of closed ideals and $a^{*}$-extensions of an abelian l-group, submitted.

2. P. F. Conrad, Archimedean extensions of lattice-ordered groups, J. Indian Math. Soc., 30 (1966), 131-160.

3. - Representation of partially ordered abelian groups as groups of real valued functions, Acta Math., 116 (1966), 199-221.

4. A. M. W. Glass, The lattice of convex directed subgroups of a directed interpolation group, Notices of the Amer. Math. Soc., 19 (1972), 72T-A94, p. A-430.

5. - Polars and their applications in directed interpolation groups, Trans. Amer. Math. Soc., 166 (1972), 1-25.

6. D. Khuon, Cardinales des groupes réticulés: complété archimedéan d'un groupe réticulé, C. R. Acad. Sci. Paris Série A, 270 (1970), 1150-1153.

7. N. R. Reilly, Compatible tight Riesz orders and prime subgroups, submitted.

8. J. R. Teller, A theorem on Riesz groups, Trans. Amer. Math. Soc., 130 (1968), 254-264.

9. K. M. Van Meter, Sur les groups *-quasi-réticulés, Pacific J. Math. (to appear).

10. A. Wirth, Compatible tight Riesz orders, J Aust. Math. Soc. (to appear).

Received October 1, 1971 and in revised form August 29, 1972.

Bowling Green State University 
